\title{
CORRELATIONS FOR TRANSIENT THERMAL CONVECTION OF WATER NEAR ITS DENSITY- INVERSION POINT IN A SQUARE ENCLOSURE HEATED FROM BELOW
}

\author{
Claudio Cianfrini, Massimo Corcione* and Alessandro Quintino \\ DIAEE Sezione Fisica Tecnica \\ Sapienza Università di Roma \\ via Eudossiana 18, 00184 Rome, Italy \\ e-mail: massimo.corcione@uniroma1.it
}

\begin{abstract}
Transient natural convection in water-filled square enclosures with the bottom wall heated at $8^{\circ} \mathrm{C}$ and the top wall cooled at $0^{\circ} \mathrm{C}$ is studied numerically for different cavity widths, in the hypothesis of temperature-dependent physical properties, assuming that the initial temperature of water is $0^{\circ} \mathrm{C}$. A computational code based on the SIMPLE-C algorithm is used to solve the system of the mass, momentum and energy transfer governing equations. The propagation of convective motion from the bottom to the top of the enclosure is investigated up to the achievement of the steady-state regime. A number of dimensionless correlations are developed for the calculations of the heat transfer rates at the heated and cooled walls during time, as well as at steady-state.
\end{abstract}

Received: January 28, 2015; Revised: February 10, 2015; Accepted: March 2, 2015

Keywords and phrases: transient natural convection, water, density-inversion point, numerical analysis, correlating equations.

${ }^{*}$ Corresponding author 


\section{Nomenclature}

$a-d \quad$ coefficients

c specific heat at constant pressure, $\mathrm{J} /(\mathrm{kg} \mathrm{K})$

g gravity vector, $\mathrm{m} / \mathrm{s}^{2}$

I unit tensor

$k$ thermal conductivity, $\mathrm{W} /(\mathrm{m} \mathrm{K})$

$\mathrm{Nu} \quad$ Nusselt number

Pr Prandtl number

$p \quad$ pressure, $\mathrm{Pa}$

$Q \quad$ heat transfer rate, $\mathrm{W}$

$q$ heat flux, $\mathrm{W} / \mathrm{m}^{2}$

$R a \quad$ Rayleigh number

$t \quad$ temperature, ${ }^{\circ} \mathrm{C}$

$U$ horizontal velocity component, $\mathrm{m} / \mathrm{s}$

V velocity vector, $\mathrm{m} / \mathrm{s}$

$V \quad$ vertical velocity component, $\mathrm{m} / \mathrm{s}$

$W \quad$ width of the enclosure, $\mathrm{m}$

$x \quad$ horizontal Cartesian coordinate, $\mathrm{m}$

$y \quad$ vertical Cartesian coordinate, $\mathrm{m}$

\section{Greek symbols}

$\varphi \quad$ generic physical property

$\mu \quad$ dynamic viscosity, $\mathrm{kg} /(\mathrm{ms})$

$\rho \quad$ mass density, $\mathrm{kg} / \mathrm{m}^{3}$

$\tau \quad$ time, $s$

$\psi \quad$ stream function, $\mathrm{kg} /(\mathrm{ms})$ 


\section{Subscripts}

$c \quad$ cooled top wall, at the temperature of the cooled top wall

$h \quad$ heated bottom wall, at the temperature of the heated bottom wall

max maximum value

$s \quad$ at steady-state

trans relevant to the transition from conduction to convection

$0 \quad$ at $0^{\circ} \mathrm{C}$

\section{Introduction}

Natural convection in a horizontal water layer differentially heated at the bottom and top boundaries with density-inversion in the bulk leads to the formation of an upper stably stratified fluid region, as well as a lower convectively unstable region from which motion can propagate upwards. This phenomenon is often referred to as penetrative convection.

Besides the studies executed on infinite horizontal layers considering either stress-free or rigid horizontal boundaries [1-6], the papers readily available in the open literature on thermal convection in enclosures filled with water near its density-inversion point are relatively few. Townsend [7] executed an experimental study using a tank whose bottom and top surfaces were kept at $0^{\circ} \mathrm{C}$ and $25^{\circ} \mathrm{C}$, respectively. The existence of a vigorous overturning in a thin water layer adjacent to the bottom wall, from which columns of buoyant fluid emerged travelling upwards up to impinging on an upper region of stable stratification and then being deflected horizontally, was detected. Later investigations were performed numerically on square cavities by Zubkov and Kalabin [8], on cubic enclosures by Zubkov et al. [9], and on shallow cylindrical containers by Li et al. [10], in all of which the heating-from-below condition was implemented in the hypothesis of constant physical properties, except for the density in the buoyancy force term of the momentum equation. Additionally, partly related studies are those recently conducted by Li and colleagues using annular enclosures having more or less 
complex configurations [11-15]. Primary aim of all these cited works was to show the existence of multiple stable steady-state or periodically-oscillating flow pattern solutions, while no special attention was devoted to the transient regime. Another study with a bearing on the subject discussed here is the numerical investigation carried out by Forbes and Cooper [16] on transient natural convection of water initially at either $4^{\circ} \mathrm{C}$ or $8^{\circ} \mathrm{C}$, confined laterally and underneath by rigid insulators, with the upper surface maintained at a constant $0^{\circ} \mathrm{C}$ temperature. Also, in this case, the physical properties were assumed as constant, incorporating the buoyancy effects by invoking the Boussinesq approximation.

Framed in this general background, a study of transient natural convection in water-filled square enclosures whose bottom and top walls are maintained at $8^{\circ} \mathrm{C}$ and $0^{\circ} \mathrm{C}$, respectively, is performed numerically for different cavity widths in the hypothesis of temperature-dependent physical properties, assuming that the initial temperature of water is $0^{\circ} \mathrm{C}$. Main scope of this work is to investigate the propagation of convective motion from the bottom to the top of the cavity, and determine the consequent time-evolution of the heat transfer rates at the heated and cooled walls up to the achievement of the steady-state regime.

\section{Mathematical Formulation}

A water-filled square enclosure of width $W$ is differentially heated at the bottom and top walls, which are maintained at uniform temperatures of $8^{\circ} \mathrm{C}$ and $0^{\circ} \mathrm{C}$, respectively, whereas both sides are assumed to be adiabatic, as shown in Figure 1, where the reference Cartesian coordinate system $(x, y)$ is also represented. The consequent buoyancy-induced flow is considered to be two-dimensional, laminar and incompressible, with negligible viscous dissipation and pressure work. 


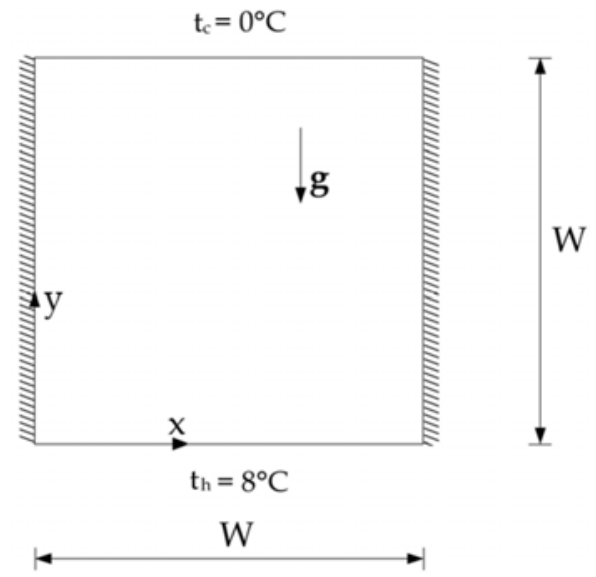

Figure 1. Sketch of the geometry and coordinate system.

In these hypotheses, the governing conservation equations of mass, momentum and energy reduce to

$$
\begin{aligned}
& \frac{\partial \rho}{\partial \tau}+\nabla \cdot(\rho \mathbf{V})=0 \\
& \frac{\partial(\rho \mathbf{V})}{\partial \tau}+\nabla \cdot(\rho \mathbf{V V})=-\nabla \cdot\left(p+\frac{2}{3} \mu \nabla \cdot \mathbf{V}\right) \mathbf{I}+\nabla \cdot \mu\left[\nabla \mathbf{V}+(\nabla \mathbf{V})^{t}\right]+\rho \mathbf{g}, \\
& \frac{\partial(\rho c t)}{\partial \tau}+\nabla \cdot(\rho \mathbf{V} c t)=\nabla \cdot(k \nabla t)
\end{aligned}
$$

where $\tau$ is the time, $\mathbf{V}$ is the velocity vector having horizontal and vertical components $U$ and $V, p$ is the pressure, $t$ is the temperature in Celsius degrees, $\mathbf{g}$ is the gravity vector, $\rho$ is the mass density, $\mu$ is the dynamic viscosity, $c$ is the specific heat at constant pressure, $k$ is the thermal conductivity, and $\mathbf{I}$ is the unit tensor. Superscript $t$ indicates the transpose of $\nabla \mathbf{V}$.

The temperature-dependence of the generic physical property $\varphi$ is approximated as a fourth-order polynomial function obtained by the best fit of eighty-one equispaced reference data in the range $0^{\circ} \mathrm{C}-8^{\circ} \mathrm{C}$ extracted from the NIST Chemistry WebBook [17], put in the form:

$$
\varphi=\varphi_{0}\left(1+a t+b t^{2}+c t^{3}+d t^{4}\right),
$$


where $\varphi_{0}$ is the value of $\varphi$ at the temperature of $0^{\circ} \mathrm{C}$, while $a, b, c$ and $d$ are the polynomial coefficients, given in Table 1. For any interpolation equation, the percentage standard deviation of the errors is of the order of $10^{-5}$. As far as the mass density is specifically concerned, its temperature-distribution is represented in Figure 2, in which the values obtained by using the correlations proposed by Gebhart and Mollendorf [18], and, more recently, by Ishikawa et al. [19], are also reported for comparison.

Table 1. Summary of the coefficients of the fourth-order polynomial functions used to quantify the temperature-dependence of the physical properties of water between $0^{\circ} \mathrm{C}$ and $8^{\circ} \mathrm{C}$

\begin{tabular}{ccccc}
\hline$\varphi$ & $\rho$ & $\mathrm{k}$ & $\mu$ & $\mathrm{c}$ \\
\hline$\varphi_{0}$ & $9.99844 \times 10^{2}$ & $5.61094 \times 10^{-1}$ & $1.79100 \times 10^{-3}$ & $4.21941 \times 10^{3}$ \\
$a$ & $6.75284 \times 10^{-5}$ & $3.37638 \times 10^{-3}$ & $-3.47372 \times 10^{-2}$ & $-8.16538 \times 10^{-4}$ \\
$b$ & $-9.01352 \times 10^{-6}$ & $3.26566 \times 10^{-6}$ & $9.32894 \times 10^{-4}$ & $2.98156 \times 10^{-5}$ \\
$c$ & $9.33050 \times 10^{-8}$ & $-2.81929 \times 10^{-7}$ & $-1.91789 \times 10^{-5}$ & $-6.42674 \times 10^{-7}$ \\
$d$ & $-8.00093 \times 10^{-10}$ & $3.22543 \times 10^{-10}$ & $2.12623 \times 10^{-7}$ & $7.25387 \times 10^{-9}$ \\
\hline
\end{tabular}

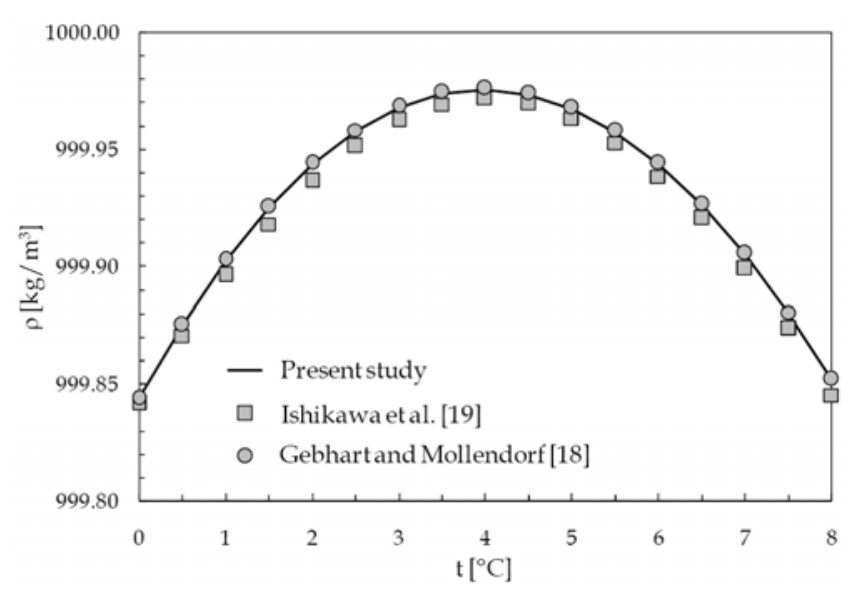

Figure 2. Comparison between the present temperature-distribution of the mass density of water near its inversion-point and two sets of values obtained using the correlations of Gebhart and Mollendorf [18] and Ishikawa et al. [19]. 
The assigned boundary conditions are: (a) $t_{h}=8^{\circ} \mathrm{C}$ and $\mathbf{V}=0$ at the bottom wall; (b) $t_{c}=0^{\circ} \mathrm{C}$ and $\mathbf{V}=0$ at the top wall; and (c) $\partial t / \partial x=0$ and $\mathbf{V}=0$ at both sidewalls.

The initial conditions assumed throughout the cavity are fluid at rest, i.e., $\mathbf{V}=0$, and a uniform temperature $t_{i}=0^{\circ} \mathrm{C}$.

\section{Computational Procedure}

The system of governing equations (1)-(3), in conjunction with the boundary and initial conditions stated earlier, is solved through a controlvolume formulation of the finite-difference method. The pressure-velocity coupling is handled through the SIMPLE-C algorithm introduced by Van Doormaal and Raithby [20], which is essentially a more implicit variant of the SIMPLE algorithm developed by Patankar and Spalding [21], whose details are thoroughly described in Patankar [22]. Convective and diffusive terms are approximated by the QUICK discretization scheme proposed by Leonard [23], whereas a second-order backward scheme is applied for timeintegration. Both space discretization and time-stepping are chosen uniform. Starting from the assigned initial fields of the dependent variables across the enclosure, at each time-step, the system of discretized algebraic governing equations is solved iteratively by way of a line-by-line application of the Thomas algorithm. A standard under-relaxation technique is enforced in all steps of the computational procedure to ensure adequate convergence. Within each time-step, the spatial numerical solution of the velocity and temperature fields is considered to be converged when the maximum absolute values of the mass source, and the relative changes of the dependent variables at any grid-node between two consecutive iterations, are smaller than the pre-

specified values of $10^{-6}$ and $10^{-7}$, respectively. Time-integration is stopped once steady-state is reached. This means that the simulation procedure ends when the relative difference between the heat transfer rates at the heated and cooled walls, and the relative changes of the time-derivatives of the dependent variables at any grid-node between two consecutive time-steps, 
are smaller than the pre-assigned values of $10^{-6}$ and $10^{-8}$, respectively. In addition, during any simulation, the dynamic behavior of the system is followed by plotting the phase trajectories of $t, U$ and $V$, at a number of assigned grid-nodes, i.e., by plotting the distributions of the time-derivatives of these variables versus the variables themselves with time as a parameter, whose attractor for steady-state solutions is represented by a fixed point.

At each time-step, after the spatial convergence is achieved, the heat fluxes at the heated and cooled walls, $q_{h}(\tau)$ and $q_{c}(\tau)$, are calculated as

$$
\begin{aligned}
& q_{h}(\tau)=-\left.k_{h} \cdot \frac{\partial t}{\partial y}\right|_{y=0}, \\
& q_{c}(\tau)=-\left.k_{c} \cdot \frac{\partial t}{\partial y}\right|_{y=w},
\end{aligned}
$$

where $k_{h}$ and $k_{c}$ are the values of the thermal conductivity of water at $t_{h}=8^{\circ} \mathrm{C}$ and $t_{c}=0^{\circ} \mathrm{C}$, respectively. The temperature gradients in equations (5) and (6) are evaluated by means of a second-order temperature profile encompassing the wall-node and the two subsequent fluid-nodes. The corresponding heat transfer rates added to the fluid by the heated bottom wall and withdrawn from the fluid by the cooled top wall, $Q_{h}(\tau)$ and $Q_{c}(\tau)$, are then quantified by the following expressions:

$$
\begin{aligned}
& Q_{h}(\tau)=\int_{0}^{W}-\left.k_{h} \cdot \frac{\partial t}{\partial y}\right|_{y=0} d x \\
& Q_{c}(\tau)=\int_{0}^{W}-\left.k_{c} \cdot \frac{\partial t}{\partial y}\right|_{y=W} d x
\end{aligned}
$$

in which the integrals are computed numerically by means of the trapezoidal rule.

The average Nusselt numbers of the heated bottom wall and the cooled top wall, $N u_{h}(\tau)$ and $N u_{c}(\tau)$, are also calculated as: 


$$
\begin{aligned}
& N u_{h}(\tau)=\frac{Q_{h}(\tau)}{k_{h} \cdot\left(t_{h}-t_{c}\right)}, \\
& N u_{c}(\tau)=\frac{Q_{c}(\tau)}{k_{c} \cdot\left(t_{c}-t_{h}\right)} .
\end{aligned}
$$

When steady-state is reached, the heat transfer rates at the heated and cooled walls, $Q_{h}$ and $Q_{c}$, coincide with the last values computed for $Q_{h}(t)$ and $Q_{c}(t)$ :

$$
Q_{\text {in }}=\left.Q_{\text {in }}(\tau)\right|_{\tau \rightarrow \infty}, \quad Q_{\text {out }}=\left.Q_{\text {out }}(\tau)\right|_{\tau \rightarrow \infty}
$$

The corresponding steady-state Nusselt numbers, $N u_{h}$ and $N u_{c}$, are given by

$$
\begin{aligned}
& N u_{h}=\frac{Q_{h}}{k_{h} \cdot\left(t_{h}-t_{c}\right)}, \\
& N u_{c}=\frac{Q_{c}}{k_{c} \cdot\left(t_{c}-t_{h}\right)} .
\end{aligned}
$$

Of course, since at steady-state the heat transfer rates at the heated and cooled walls are the same, which means that $Q_{h}=-Q_{c}=Q$, the following relationship between the steady-state Nusselt numbers holds:

$$
N u_{h} \cdot k_{h}=N u_{c} \cdot k_{c} .
$$

Numerical tests related to the dependence of the results obtained on the mesh spacing and time-stepping have been performed for several values of $W$. The discretization grids and time-steps used for computations are chosen in such a way that further refinements do not produce noticeable modifications either in the heat transfer rates or in the flow fields at steadystate. In particular, the percentage change of the heat transfer rate $Q_{h}$, as well as that of the maximum vertical velocity component $V_{\max }$ on the horizontal midplane of the enclosure, must be smaller than the preestablished accuracy value of $1 \%$. The typical number of nodal points and time-step used for simulations lie in the ranges between $120 \times 120$ and 
$180 \times 180$, and between $5 \times 10^{-3} \mathrm{~s}$ and $5 \times 10^{-2} \mathrm{~s}$, respectively. Selected results of the grid sensitivity analysis are presented in Table 2, in which the steady-state values of $Q_{h}$ and $V_{\max }$ relative to consecutive mesh sizes are reported for $W=0.04 \mathrm{~m}$ and $0.06 \mathrm{~m}$, showing that a denser grid is required at larger widths of the enclosure. As far as the time-stepping is concerned, its effects are displayed in Table 3 for $W=0.04 \mathrm{~m}$, showing that $\Delta \tau=10^{-2} \mathrm{~s}$ represents a good compromise between solution accuracy and computation time.

Table 2. Grid sensitivity analysis for $W=0.04 \mathrm{~m}$ and $W=0.06 \mathrm{~m}$

\begin{tabular}{|c|c|c|c|c|c|c|}
\hline$\Delta \tau[\mathrm{s}]$ & $\mathrm{W}[\mathrm{m}]$ & mesh size & $\mathrm{Q}_{\mathrm{h}}[\mathrm{W}]$ & $\%$ & $\mathrm{~V}_{\max }\left(\times 10^{3}\right)[\mathrm{m} / \mathrm{s}]$ & $\%$ \\
\hline \multirow[t]{11}{*}{$10^{-2}$} & 0.04 & $60 \times 60$ & 20.54 & - & 0.499 & - \\
\hline & & $80 \times 80$ & 20.32 & -1.07 & 0.508 & 1.80 \\
\hline & & $100 \times 100$ & 20.12 & -0.98 & 0.514 & 1.18 \\
\hline & & $120 \times 120$ & 20.11 & -0.05 & 0.517 & 0.58 \\
\hline & & $140 \times 140$ & 20.11 & 0.00 & 0.517 & 0.00 \\
\hline & 0.06 & $60 \times 60$ & 20.64 & - & 0.499 & - \\
\hline & & $80 \times 80$ & 20.36 & -1.36 & 0.515 & 3.21 \\
\hline & & $100 \times 100$ & 20.15 & -1.03 & 0.526 & 2.14 \\
\hline & & $120 \times 120$ & 20.11 & -0.20 & 0.532 & 1.14 \\
\hline & & $140 \times 140$ & 20.11 & 0.00 & 0.529 & -0.56 \\
\hline & & $160 \times 160$ & 20.11 & 0.00 & 0.528 & -0.19 \\
\hline
\end{tabular}

Table 3. Time-step sensitivity analysis for $W=0.04$

\begin{tabular}{ccccccc}
\hline $\mathrm{W}[\mathrm{m}]$ & mesh size & $\Delta \tau[\mathrm{s}]$ & $\mathrm{Q}_{\mathrm{h}}[\mathrm{W}]$ & $\%$ & $\mathrm{~V}_{\max }\left(\times 10^{3}\right)[\mathrm{m} / \mathrm{s}]$ & $\%$ \\
\hline 0.04 & $120 \times 120$ & $10^{0}$ & 20.29 & - & 0.528 & - \\
& & $10^{-1}$ & 20.15 & -0.69 & 0.521 & -1.33 \\
& & $10^{-2}$ & 20.11 & -0.20 & 0.517 & -0.77 \\
& & $10^{-3}$ & 20.10 & -0.05 & 0.517 & 0.00 \\
\hline
\end{tabular}


Finally, with the aim to validate the numerical code used for the present study, three different tests have been carried out. In the first test, the steadystate solutions computed numerically for an air-filled square enclosure differentially heated at sides by assuming constant physical properties have been compared with the benchmark results obtained by de Vahl Davis [24] through a standard finite-difference method, as shown in Table 4.

Table 4. Comparison of the present numerical results and the benchmark solutions of de Vahl Davis [24], Mahdi and Kinney [25], Hortmann et al. [26] and Wan et al. [27], for an air-filled differentially heated square cavity at steady-state

\begin{tabular}{|c|c|c|c|c|c|}
\hline Quantities & Present work & BM1 & BM2 & BM3 & BM4 \\
\hline \multicolumn{6}{|c|}{$\mathrm{Ra}=10^{3}$} \\
\hline $\mathrm{u}_{\max }$ & 3.654 & 3.649 & 3.649 & 3.489 & 3.643 \\
\hline $\mathrm{v}_{\max }$ & 3.708 & 3.697 & 3.690 & 3.686 & 3.686 \\
\hline $\mathrm{Nu}$ & 1.116 & 1.118 & 1.113 & 1.117 & 1.073 \\
\hline \multicolumn{6}{|c|}{$\mathrm{Ra}=10^{4}$} \\
\hline $\mathrm{u}_{\max }$ & 16.242 & 16.178 & 16.180 & 16.122 & 15.967 \\
\hline $\mathrm{v}_{\max }$ & 19.714 & 19.617 & 19.629 & 19.790 & 19.980 \\
\hline $\mathrm{Nu}$ & 2.254 & 2.243 & 2.244 & 2.254 & 2.155 \\
\hline \multicolumn{6}{|c|}{$\mathrm{Ra}=10^{5}$} \\
\hline $\mathrm{u}_{\max }$ & 35.008 & 34.730 & 34.739 & 33.390 & 33.510 \\
\hline $\mathrm{V}_{\max }$ & 68.109 & 68.590 & 68.639 & 70.630 & 70.810 \\
\hline $\mathrm{Nu}$ & 4.506 & 4.519 & 4.521 & 4.598 & 4.352 \\
\hline \multicolumn{6}{|c|}{$\mathrm{Ra}=10^{6}$} \\
\hline $\mathrm{u}_{\max }$ & 65.226 & 64.630 & 64.836 & 65.400 & 65.550 \\
\hline $\mathrm{v}_{\max }$ & 221.598 & 219.360 & 220.461 & 227.110 & 227.240 \\
\hline $\mathrm{Nu}$ & 8.879 & 8.800 & 8.825 & 8.976 & 8.632 \\
\hline
\end{tabular}

$\mathrm{BMl}=$ de Vahl Davis [24]

BM2 $=$ Mahdi and Kinney [25] for Ra $=10^{3}$, and Hortman et al. [26] for Ra $=10^{4}-10^{6}$

BM3 $=$ Wan et al. - FEM [27]

BM4 $=$ Wan et al. - DSC [27] 
It may be seen that the average Nusselt number, $N u$, and the maximum horizontal and vertical dimensionless velocity components on the vertical and horizontal midplanes of the cavity, $u_{\max }$ and $v_{\max }$, are well within $1 \%$ of the benchmark data listed in column BM1. The following extra benchmark solutions are also enumerated for further comparison: (a) the results obtained through finite-volume methods by Mahdi and Kinney [25] for $R a=10^{3}$ and by Hortmann et al. [26] for $R a=10^{4}-10^{6}$ are listed in column BM2; (b) the results obtained through a finite-element method by Wan et al. [27] are listed in column BM3; and (c) the results obtained by means of a discrete singular convolution algorithm by Wan et al. [27] are listed in column BM4. In the second test, the values of the average Nusselt number at steady-state obtained for $\operatorname{Pr}=7$ and $R a=10^{3}-5 \times 10^{7}$ have been compared with the usually recommended Berkovsky-Polevikov correlation based on both experimental and numerical data of laminar natural convection in a rectangular cavity heated and cooled from the side with an aspect ratio near unity, see, e.g., Bejan [28] and Incropera et al. [29]. The comparative analysis, displayed in Figure 3, demonstrates that the correspondence between numerical results and literature data is widely satisfactory.

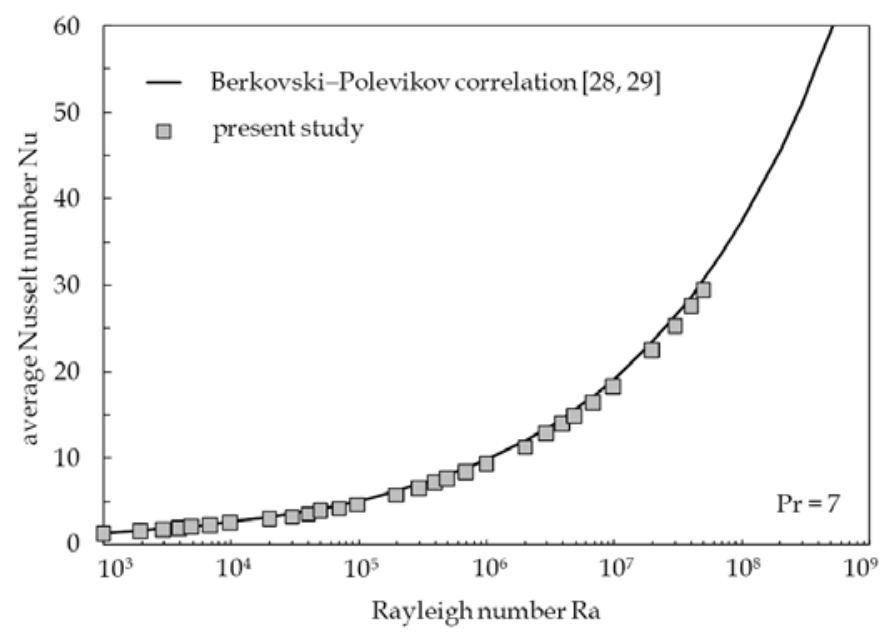

Figure 3. Comparison between the present numerical results and the Berkovski-Polevikov correlation for a water-filled enclosure differentially heated at sides. 
In the third test, the steady-state solutions obtained in the hypothesis of variable physical properties for a water-filled square cavity whose sides are differentially heated at temperatures of $0^{\circ} \mathrm{C}$ and $8^{\circ} \mathrm{C}$, have been compared with the numerical results of Ishikawa et al. [19], as depicted in Figure 4. Also, in this case, a very good compliance between numerical results and literature data is apparent.

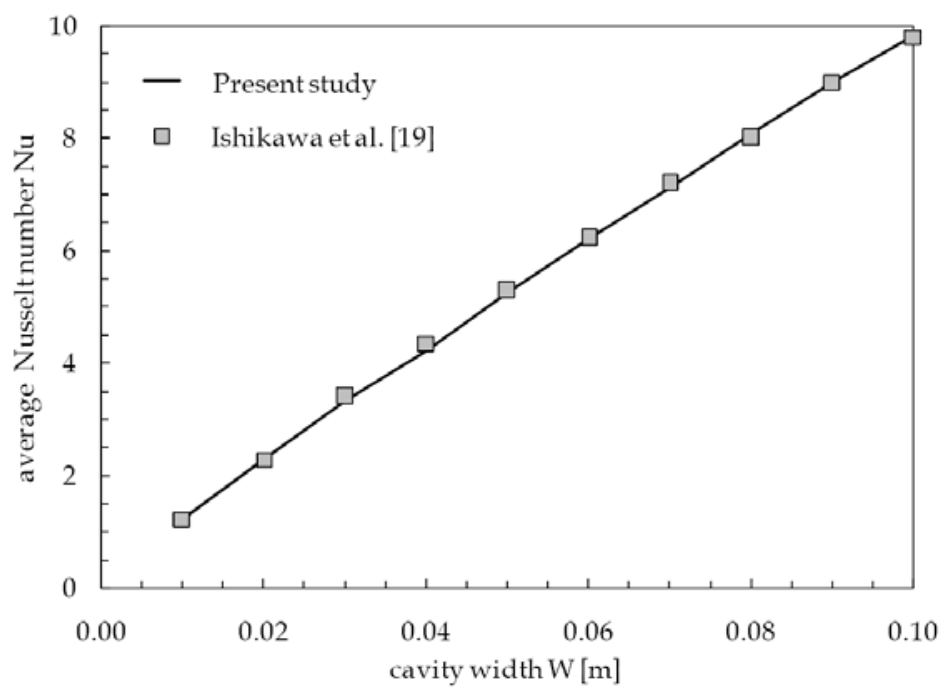

Figure 4. Comparison between the present numerical results and the numerical data of Ishikawa et al. [19] for a water-filled square enclosure whose sides are differentially heated at $8^{\circ} \mathrm{C}$ and $0^{\circ} \mathrm{C}$ in the hypothesis of temperature-dependent physical properties.

\section{Results and Discussion}

Numerical simulations are performed for different values of the cavity width, $W$, in the range between $3 \mathrm{~cm}$ and $7 \mathrm{~cm}$, using an investigation step of $0.5 \mathrm{~cm}$. The corresponding Rayleigh numbers are of the order of $10^{5}-10^{6}$. 

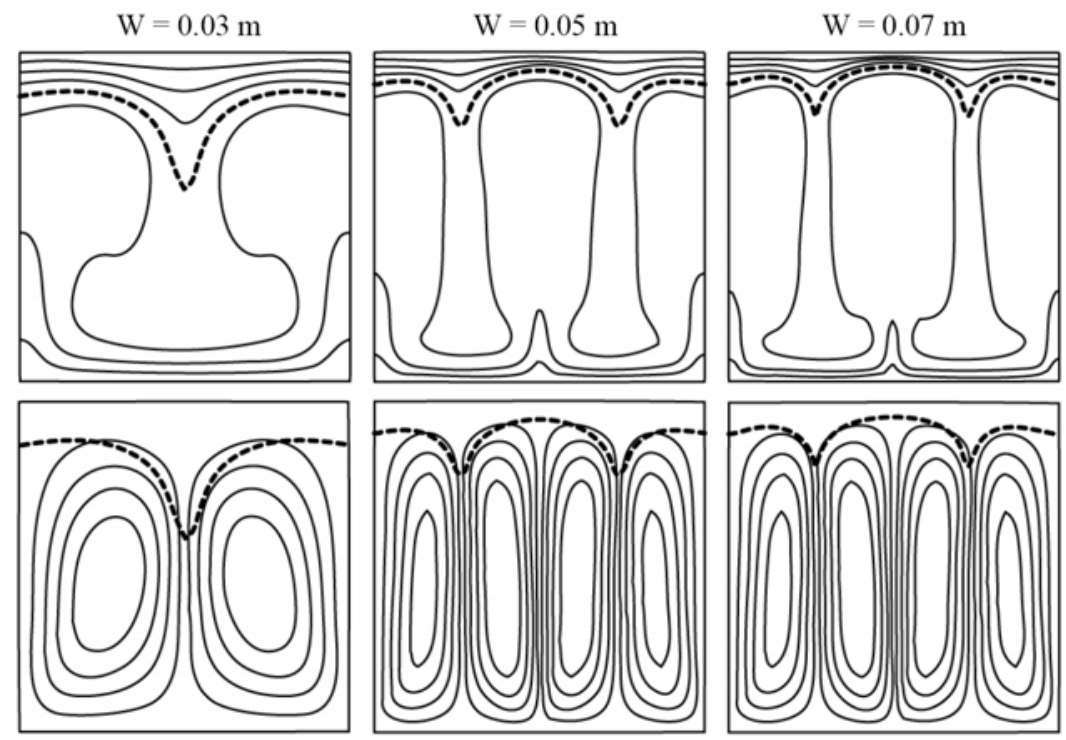

$|\psi|_{\max }=2.54 \times 10^{-3} \mathrm{~kg} /(\mathrm{ms})$

$|\psi|_{\max }=2.67 \times 10^{-3} \mathrm{~kg} /(\mathrm{ms})$

$|\psi|_{\max }=5.06 \times 10^{-3} \mathrm{~kg} /(\mathrm{ms})$

Figure 5. Isotherm and streamline contour plots at steady-state for $W=$ $0.03 \mathrm{~m}, 0.05 \mathrm{~m}$ and $0.07 \mathrm{~m}$.

A selection of local results at steady-state is presented in Figure 5 in the form of isotherm and streamline contour plots for $W=0.03 \mathrm{~m}, 0.05 \mathrm{~m}$ and $0.07 \mathrm{~m}$, showing that the flow field consists of counter-rotating cells whose number increases with increasing the width of the enclosure. The contours of the isotherm plots correspond to equally-spaced temperatures in the range between $0^{\circ} \mathrm{C}$ and $8^{\circ} \mathrm{C}$. The contours of the streamline plots correspond to equally-spaced absolute values of the stream function $\psi$ in the range between 0 and $|\psi|_{\max }$, where $\psi$ is defined as usual by $\rho U=\partial \psi / \partial y$ and $\rho V=-\partial \psi / \partial x$. The $4^{\circ} \mathrm{C}$ isotherm, represented using a dashed line, is superimposed on any streamline pattern, in order to give an indication of the effect of the maximum density on the flow field. The heat transfer performance of the enclosure at steady-state, expressed in terms of the average Nusselt number of the heated bottom wall, $N u_{h}$, as well as the dimensionless time needed to arrive at steady-state, $T_{S}$, can be correlated to the Rayleigh number, $R a$, by the following equations, as shown in Figure 6: 


$$
\begin{aligned}
& N u_{h}=0.176 R a^{0.26}, \\
& T_{S}=51.8 R a^{-0.237},
\end{aligned}
$$

where $R a$ and $T_{S}$ are defined as

$$
\begin{aligned}
& R a=\frac{c \rho g \Delta \rho W^{3}}{\mu k}, \\
& T_{s}=\frac{\tau_{s} \mu}{W^{2} \rho},
\end{aligned}
$$

in which $\Delta \rho$ is the difference between the water densities at $4{ }^{\circ} \mathrm{C}$ and $0^{\circ} \mathrm{C}$, while all the other physical properties are evaluated at the density-inversion temperature of $4^{\circ} \mathrm{C}$. The standard deviations of the errors are $1.8 \%$ for equation (12), and $2.2 \%$ for equation (13). Once the value of $N u_{h}$ is known, the average Nusselt number of the cooled top wall at steady-state, $N u_{c}$, can be calculated using equation (11).

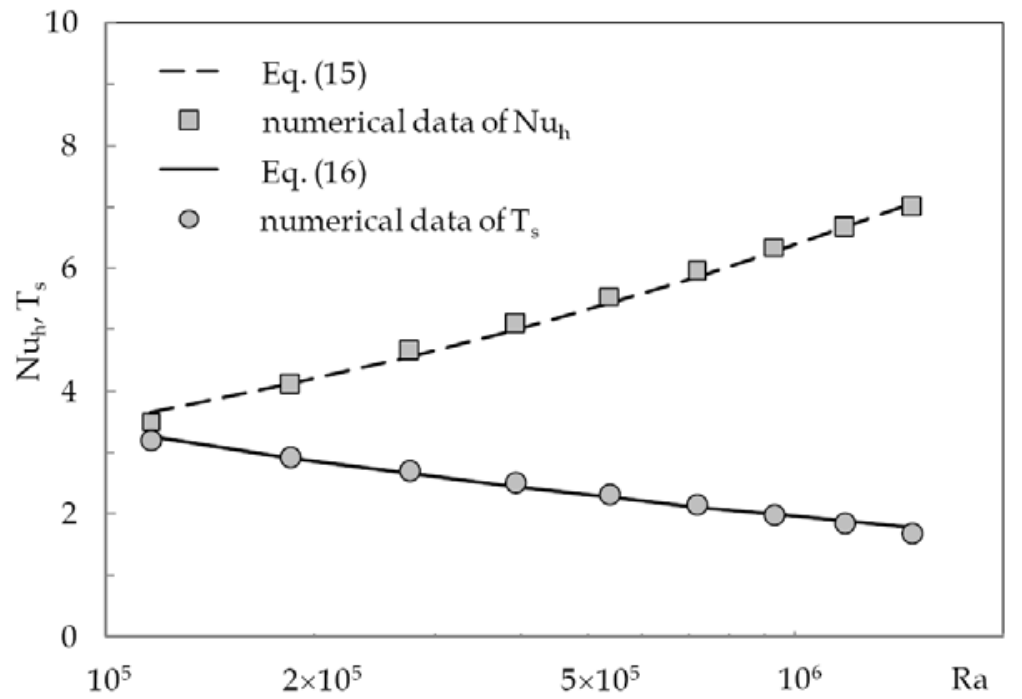

Figure 6. Comparison between equations (15) and (16) and the numerical data. 

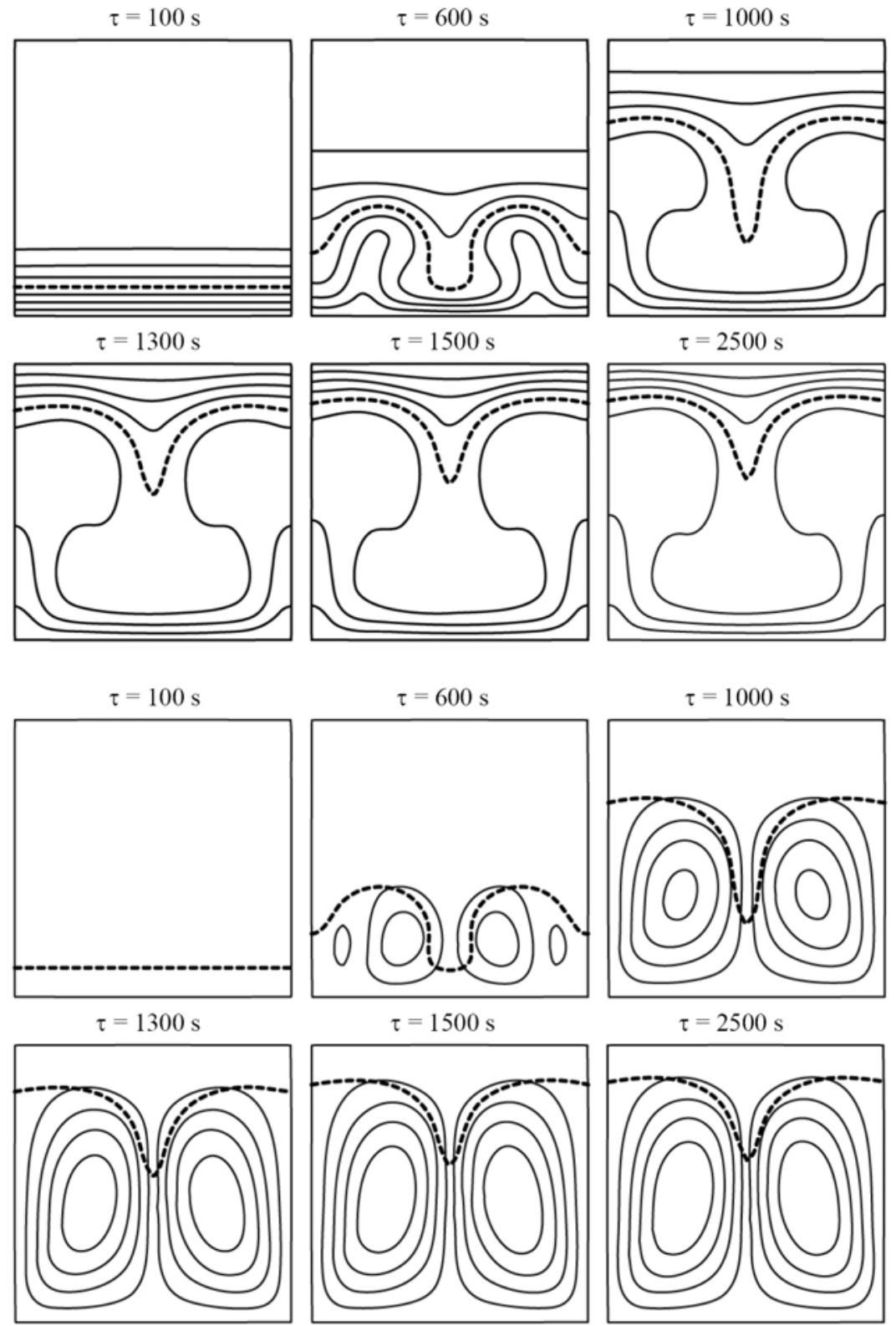

Figure 7. Time-evolution of the isotherm and streamline contour plots for $W=0.03 \mathrm{~m}$. 

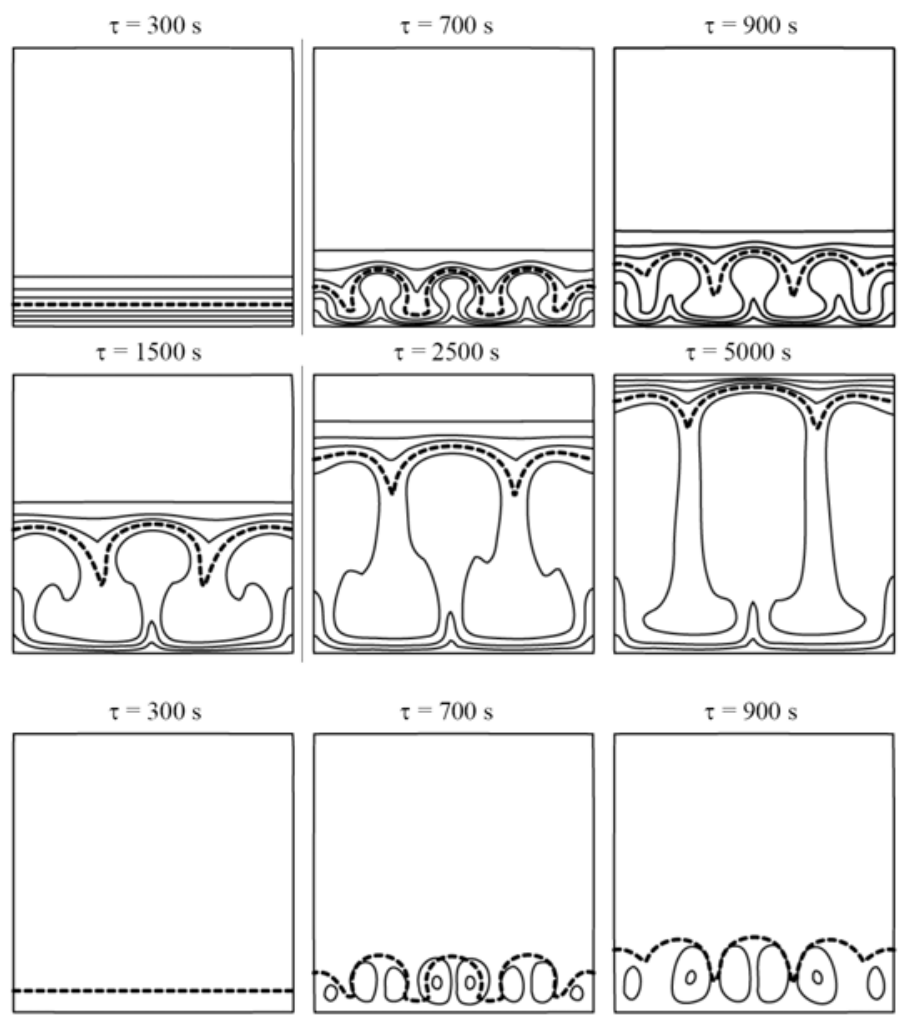

$\tau=1500$

$\tau=2500 \mathrm{~s}$

$\tau=5000 \mathrm{~s}$
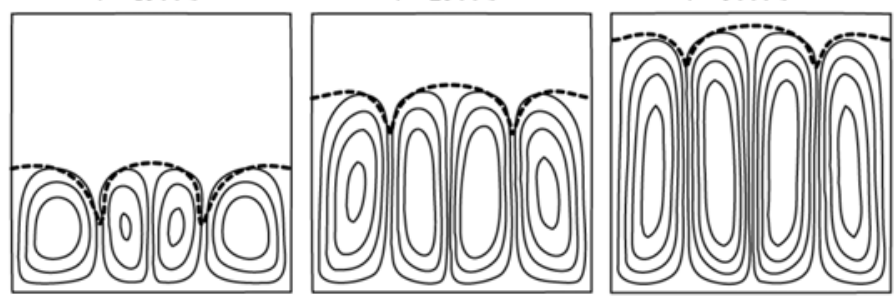

Figure 8. Time-evolution of the isotherm and streamline contour plots for $W=0.07 \mathrm{~m}$.

The time-evolutions of the temperature and flow fields for $W=0.03 \mathrm{~m}$ and $0.07 \mathrm{~m}$ are documented in Figures 7 and 8 , respectively, through a sequence of six snapshots, in which the isotherm and streamline contours are plotted at subsequent times. The corresponding time-distributions of the heat transfer rates $Q_{h}(\tau)$ and $Q_{c}(\tau)$ at the bottom and top walls are depicted in Figures 9 and 10, in which, for the sake of clarity, only a very limited 
number of the available numerical data are actually displayed. It is worth observing that the step discontinuity of the distributions of $Q_{h}(\tau)$ corresponds to the transition from the conduction dominated regime to the convection dominated regime, which occurs as soon as the $4^{\circ} \mathrm{C}$ isotherm travels at a distance from the bottom heated wall such that the Rayleigh number of the underlying water layer reaches the critical value for the onset of convection. As depicted in Figures 7 and 8, after their formation, the roll-cells merge and develop gradually from bottom to top, penetrating the water stratification that tends to establish in the upper portion of the enclosure. Of course, the thicker is the stratified water layer to be penetrated, the longer is the time required for the motion to propagate upwards to the top of the enclosure, and then the achievement of the steady-state regime. Notice that the four-cell flow structure found for the enclosures having a width $W \geq 0.045 \mathrm{~m}$, and then a Rayleigh number higher than $4 \times 10^{5}$, is in full line with the steady-state flow pattern previously found by Zubkov and Kalabin [8] for similar Rayleigh numbers. Actually, two points have to be cleared up in respect with this matter. First, Zubkov and Kalabin reported the presence of two pairs of secondary cells located in the top region of the enclosure. Indeed, we also discovered their existence, yet these secondary vortices are not depicted in our equally-spaced representation of the streamlines as the corresponding values of the stream function $\psi$ are of the order of $10^{-5}-10^{-6} \mathrm{~kg} /(\mathrm{ms})$, i.e., two orders of magnitude lower than those relevant to the main circulation. Secondly, according to Zubkov and Kalabin, the four-cell flow structure appears at a critical Rayleigh number of nearly $3.3 \times 10^{5}$. Conversely, as said earlier, we detected the development of such a flow pattern for $W \geq 0.045 \mathrm{~m}$, i.e., for $R a \geq 4 \times 10^{5}$. Indeed, this can be explained by considering that, since we conducted our study using an investigation step of $0.5 \mathrm{~cm}$, the value $R a=4 \times 10^{5}$ must not be intended as a critical value, but rather as the first value of the Rayleigh number at which we obtained a flow configuration consisting of four primary cells (notice that the Rayleigh number corresponding to $W=0.04 \mathrm{~m}$ is approximately $2.76 \times 10^{5}$ ). 
Correlations for Transient Thermal Convection of Water ...

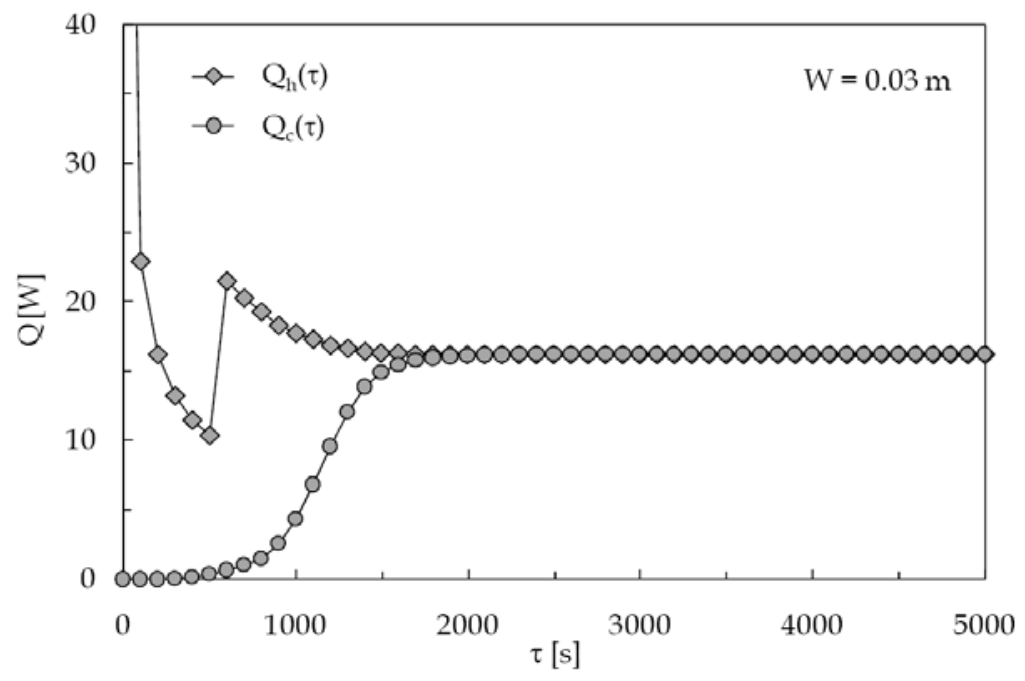

Figure 9. Time-evolution of the heat transfer rates at the heated bottom wall and the cooled top wall for $W=0.03 \mathrm{~m}$.

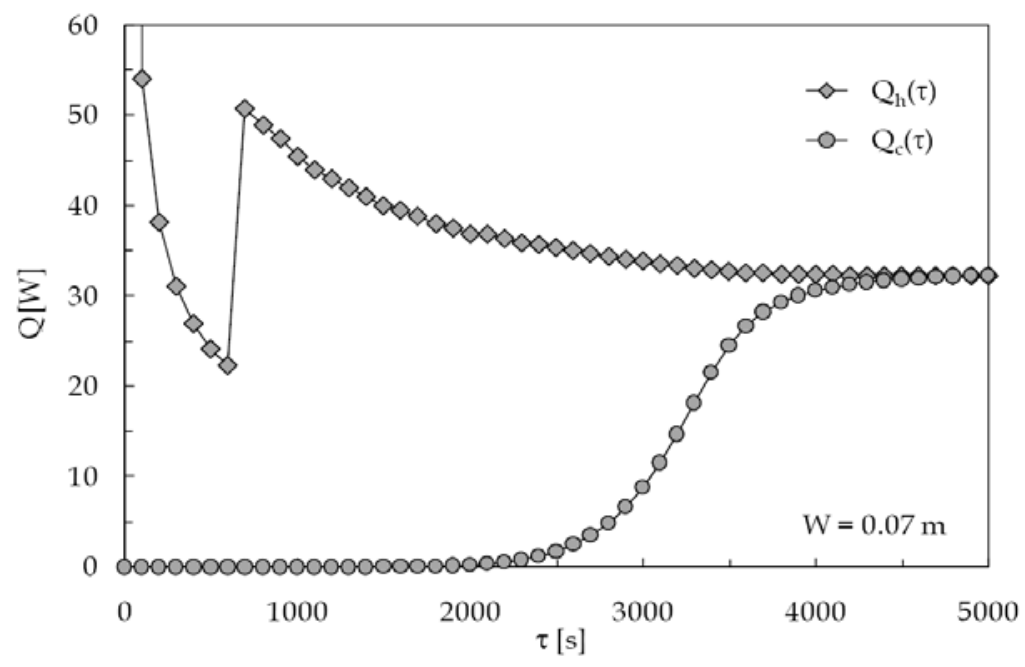

Figure 10. Time-evolution of the heat transfer rates at the heated bottom wall and the cooled top wall for $W=0.07 \mathrm{~m}$. 
For all the configurations investigated, the distributions of $N u_{h}(\tau)$ and $N u_{c}(\tau)$, normalized using the relevant steady-state values $N u_{h}$ and $N u_{c}$, can be correlated to the dimensionless time $T=\tau \mu / W^{2} \rho$, normalized using the related dimensionless time needed to arrive at steady-state $T_{S}$, by the following empirical equations, as displayed in Figures 11 and 12, respectively:

$$
\begin{aligned}
& \frac{N u_{h}(\tau)}{N u_{h}}=0.312\left(T / T_{S}\right)^{-0.43} \text { for } 0<\left(T / T_{S}\right) \leq\left(T_{\text {trans }} / T_{S}\right), \\
& \frac{N u_{h}(\tau)}{N u_{h}}=1+0.8\left[1-\left(T / T_{S}\right)\right]^{3.6} \text { for }\left(T_{\text {trans }} / T_{S}\right)<\left(T / T_{S}\right) \leq 1, \\
& \frac{N u_{c}(\tau)}{N u_{c}}=0.5\left\{1+\tanh \left[9.4\left(T / T_{S}\right)-5.6\right]\right\} \text { for } 0 \leq\left(T / T_{S}\right) \leq 1,
\end{aligned}
$$

wherein $\left(T_{\text {trans }} / T_{S}\right)$ is the normalized dimensionless time at which the transition from the conduction dominated regime to the convection dominated regime takes place, whose value is correlated to the Rayleigh number by

$$
\left(T_{\text {trans }} / T_{S}\right)=23.7 R a^{-0.386}
$$

The standard deviations of the errors of the preceding equations are $4.8 \%$ for equation (19), 3.1\% for equation (20), 3.8\% for equation (21), and $2.8 \%$ for equation (22). Thus, once the values of $N u_{h}, N u_{c}$ and $T_{s}$, calculated through equations (14)-(16), are substituted into equations (19)-(22), and the resulting values of $N u_{h}(\tau)$ and $N u_{c}(\tau)$ are then replaced in equations (9) and (10), the time-distributions of the heat transfer rates $Q_{h}(\tau)$ and $Q_{c}(\tau)$ at the bottom and top walls of the cavity, up to the achievement of the steadystate regime, are completely defined. 


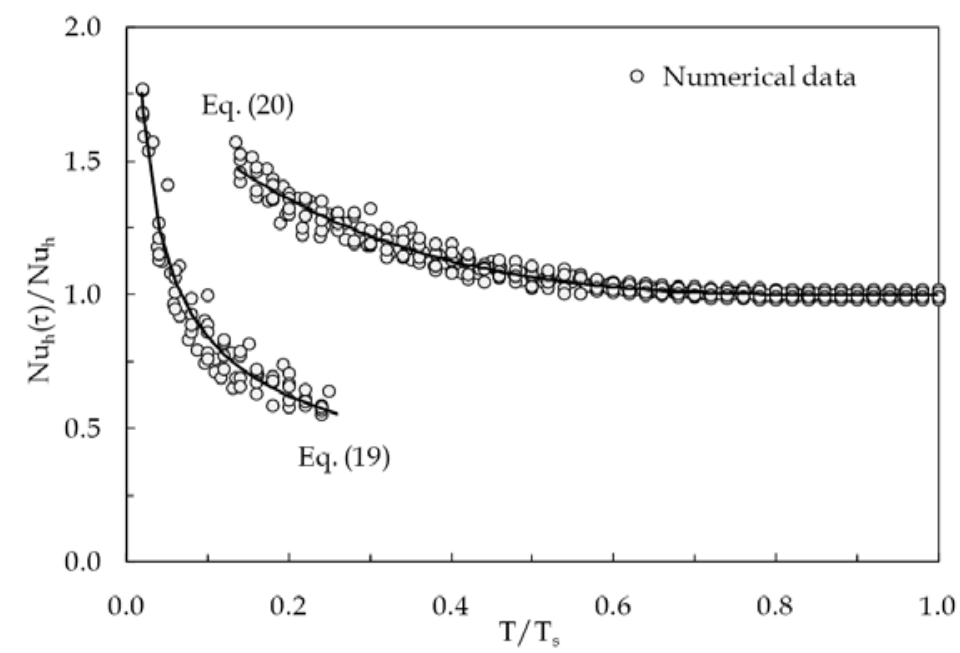

Figure 11. Comparison between equations (19) and (20) and the distributions of the normalized average Nusselt number of the bottom heated wall plotted against the normalized dimensionless time for all the configurations investigated.

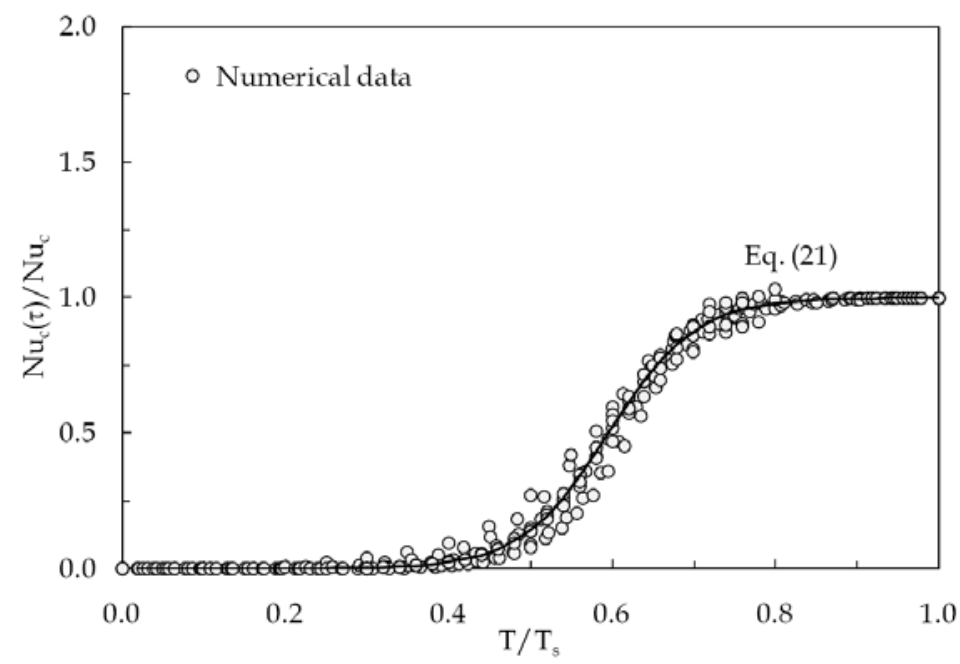

Figure 12. Comparison between equation (21) and the distributions of the normalized average Nusselt number of the top cooled wall plotted against the normalized dimensionless time for all the configurations investigated. 


\section{Conclusions}

Transient natural convection of water in square enclosures with the bottom wall heated at $8^{\circ} \mathrm{C}$ and the top wall cooled at $0^{\circ} \mathrm{C}$ has been investigated numerically, in the hypothesis of temperature-dependent physical properties, starting from the initial condition of motionless fluid at $0^{\circ} \mathrm{C}$. The system of the mass, momentum and energy transfer governing equations has been solved by a computational code based on the SIMPLE-C algorithm. Simulations have been performed for different cavity widths in the range between $0.03 \mathrm{~m}$ and $0.07 \mathrm{~m}$.

It has been found that in the first time interval from the beginning, a conduction dominated regime prevails. Soon after the $4^{\circ} \mathrm{C}$ isotherm reaches a distance from the bottom wall such that the buoyancy force in the underlying water layer is sufficiently strong, a transition to a convection dominated regime occurs. Subsequently, the convective motion propagates from the bottom of the cavity to the top, penetrating the overlying stratified water, up to the achievement of a steady-state flow structure. Accordingly, a set of dimensionless correlating equations has been proposed to describe the time evolutions of the heat transfer rates at the heated and cooled horizontal walls of the enclosure.

\section{References}

[1] G. Veronis, Penetrative convection, Astrophys. J. 137 (1963), 641-663.

[2] S. Musman, Penetrative convection, J. Fluid Mech. 31 (1968), 343-360.

[3] D. R. Moore and N. O. Weiss, Nonlinear penetrative convection, J. Fluid Mech. 61 (1973), 553-581.

[4] D. V. Kuznetsova and I. N. Sibgatullin, Transitional regimes of penetrative convection in a plane layer, Fluid Dyn. Res. 44 (2012), 031410.

[5] K. R. Blake, D. Poulikakos and A. Bejan, Natural convection near $4^{\circ} \mathrm{C}$ in a horizontal water layer heated from below, Phys. Fluids 27 (1984), 2608-2616. 
[6] W. Tong and J. N. Koster, Penetrative convection in sublayer of water including density inversion, Wärme and Stoffübertragung 29 (1993), 37-49.

[7] A. A. Townsend, Natural convection in water over an ice surface, Quart. J. Roy. Metereol. Soc. 90 (1964), 248-259.

[8] P. T. Zubkov and E V. Kalabin, Numerical investigation of the natural convection of water in the neighborhood of the density inversion point for Grashof numbers up to 106, Fluid Dyn. 36 (2001), 944-951.

[9] P. T. Zubkov, E V. Kalabin and A. V. Yakovlev, Investigation of natural convection in a cubic cavity near $4^{\circ} \mathrm{C}$, Fluid Dyn. 37 (2002), 847-853.

[10] Y. R. Li, Y. Q. Ouyang and Y. P. Hu, Pattern formation of Rayleigh-Bénard convection of cold water near its density maximum in a vertical cylindrical container, Phys. Rev. E 86 (2012), 046323.

[11] Y. R. Li, X. F. Yuan, C. M. Wu and Y. P. Hu, Natural convection of water near its density maximum between horizontal cylinders, Int. J. Heat Mass Transfer 54 (2011), 2550-2559.

[12] Y. R. Li, Y. P. Hu and X. F. Yuan, Natural convection of water near its density maximum around a cylinder inside an elliptical enclosure along slender orientation, Num. Heat Transfer A 62 (2012), 780-797.

[13] Y. R. Li, Y. P. Hu and X. F. Yuan, Three-dimensional numerical simulation of natural convection of water near its density maximum in a horizontal annulus, Int. J. Thermal Sciences 71 (2013), 274-282.

[14] Y. P. Hu, Y. R. Li, X. F. Yuan and C. M. Wu, Natural convection of cold water near its density maximum in an elliptical enclosure containing a coaxial cylinder, Int. J. Heat Mass Transfer 60 (2013), 170-179.

[15] Y. P. Hu, Y. R. Li and C. M. Wu, Comparison investigation on natural convection of cold water near its density maximum in annular enclosures with complex configurations, Int. J. Heat Mass Transfer 72 (2014), 572-584.

[16] R. E. Forbes and J. W. Cooper, Natural convection in a horizontal layer of water cooled from above to near freezing, J. Heat Transfer - Trans. ASME 97 (1975), 47-53.

[17] P. J. Linstrom and W. G. Mallard, eds., NIST Chemistry WebBook, NIST Standard Reference Database Number 69, National Institute of Standards and Technology, Gaithersburg MD, 20899, USA, http://webbook.nist.gov. 
88 Claudio Cianfrini, Massimo Corcione and Alessandro Quintino

[18] B. Gebhart and J. C. Mollendorf, A new density relation for pure and saline water, Deep-Sea Research 24 (1977), 831-848.

[19] M. Ishikawa, T. Hirata and S. Noda, Numerical simulation of natural convection with density inversion in a square cavity, Num. Heat Transfer A 37 (2000), 395-406.

[20] J. P. Van Doormaal and G. D. Raithby, Enhancements of the simple method for predicting incompressible fluid flows, Num. Heat Transfer 7 (1984), 147-163.

[21] S. V. Patankar and D. B. Spalding, A calculation procedure for heat, mass and momentum transfer in three-dimensional parabolic flows, Int. J. Heat Mass Transfer 15 (1972), 1787-1797.

[22] S. V. Patankar, Numerical Heat Transfer and Fluid Flow, Hemisphere Publ. Co., Washington DC, 1980.

[23] B. P. Leonard, A stable and accurate convective modelling procedure based on quadratic upstream interpolation, Comput. Methods Appl. Mech. Engrg. 19 (1979), 59-78.

[24] G. de Vahl Davis, Natural convection of air in a square cavity: a benchmark numerical solution, Internat. J. Numer. Methods Fluids 3 (1983), 249-264.

[25] H. S. Mahdi and R. B. Kinney, Time-dependent natural convection in a square cavity: application of a new finite volume method, Internat. J. Numer. Methods Fluids 11 (1990), 57-86.

[26] M. Hortmann, M. Peric and G. Scheuerer, Finite volume multigrid prediction of laminar natural convection: bench-mark solutions, Internat. J. Numer. Methods Fluids 11 (1990), 189-207.

[27] D. C. Wan, B. S. V. Patnaik and G. W. Wei, A new benchmark quality solution for the buoyancy-driven cavity by discrete singular convolution, Num. Heat Transfer 40 (2001), 199-228.

[28] A. Bejan, Convection Heat Transfer, 3rd ed., John Wiley \& Sons, Inc., Hoboken, New Jersey, 2004.

[29] F. P. Incropera, D. P. Dewitt, T. L. Bergman and A. S. Lavine, Fundamentals of Heat and Mass Transfer, 6th ed., John Wiley \& Sons, Inc., Hoboken, New Jersey, 2007. 